\title{
CCD IMAGING OF NEARBY AGED PLANETARY NEBULAE
}

\author{
J. PAPAMASTORAKIS, K.M. XILOURIS and E.V. PALEOLOGOU \\ University of Crete Physics Department \\ Heraklion 714 09, Crete, Greece, and \\ Foundation for Research Technology-Hellas \\ Heraklion 711 10, Crete, Greece
}

\begin{abstract}
We present results from narrow band CCD images in $\mathrm{H} \alpha+[\mathrm{N} \mathrm{II}]$ and [O III] of very faint, nearby (less than $0.5 \mathrm{kpc}$ ) planetary nebulae (PNe), of large angular extent.

The instrumental set up used (Mount Skinakas Wide Field Camera) is capable of detecting structures of large angular extent as faint as $10^{-17}$ ergs $\sec ^{-1} \mathrm{~cm}^{-2} \operatorname{arcsec}^{-2}$.

New faint structures are revealed mostly at large distances from the suspected central star of the PNe studied. These structures are atypical of the PNe population and hard to reconcile with the interacting stellar wind evolutionary scenario. They are rather reminiscent of the interaction of the former Red Giant Envelope with the ambient Interstellar Medium.

The characteristics of this aged sample are asymmetric emission distribution, displacement of the central star, extremely low expansion velocities, concentrations along the stellar motion, with the $[\mathrm{O}$ III] emission amorphous and confined close to the center of the PN while the $\mathrm{H} \alpha+[\mathrm{N} \mathrm{III}]$ emission is structured and more extended.
\end{abstract}

\section{Introduction}

Large-scale studies of PNe have been carried out with various methods, and numerous attempts have been made to find a general classification scheme using physical or empirical criteria. Those studies (i.e. Balick 1987; Chu et al. 1987) were restricted to nebular sizes of less than 0.4 pc. In this work we study nearby PNe which are of large angular extent and are not well studied both due to their large size and their faintness. The combination of a telescope with a wide fieldof-view together with a CCD camera facilitated ideally this study.

We selected those nebulae with large angular size and low expansion velocities as representatives of the aged PNe sample in the solar neighborhood. The signature of the expected shapes of PNe as delineated by various evolutionary scenarios (Balick 1987; Chu et al. 1987) was searched on our images.

We used our images together with kinematic data as well as data of the central star (when available) to identify the predicted signatures of interaction with the ambient ISM (Borkowski et al. 1990). Such an interaction is thought to take place at the evolutionary stage of the nebula, when its density has dropped below a certain value due to its expansion. 


\section{Instrumentation}

The observations were performed with the $30 \mathrm{~cm}$ Schmidt-Cassegrain telescope on Mount Skinakas Observatory, Crete, Greece during the period August 3 to 281992 . This observatory is a joint collaboration of the University of Crete, the Max-Planck Institut für Extraterrestrische Physik and the Foundation for Research and Technology-Hellas. The images were obtained by means of a cryogenically cooled CCD camera equipped with a TI 4849 chip. One pixel corresponds to 4.93" on the sky, giving a total field-of-view of $32^{\prime} \times 48^{\prime}$. Two interference filters have been used: $\mathrm{H} \alpha+[\mathrm{N} \mathrm{II}],(\lambda=6570, \Delta \lambda=75 \AA$ FWHM $)$ and [O III] $(\lambda=5007, \Delta \lambda=25 \AA$ $F W H M$ ). There were several exposures per PN, which were later added together during the data analysis process.

As most of the PNe are located in heavily crowded areas and in order to identify the crucial features, an important intermediate step was to subtract as completely as possible the projected stellar images. The advantage of the wide field-of-view that is offered by our instrumental set-up is compensated by the narrow PSF of the stellar images. Careful application of DAOPHOT II software running under MIDAS, produced satisfactory results in most cases. Satisfactory stellar subtraction results were also obtained by applying a modification of the COSMIC RAY detection and removal - MIDAS procedure.

Each frame, after the procedure described above, was calibrated into flux units (ergs $\mathrm{sec}^{-1} \mathrm{~cm}^{-2} \operatorname{arcsec}^{-2}$ ) above the Earth's atmosphere. The calibration was based on observations through the same filter set of the spectrophotometric standard star BD $28^{\circ} 4211$ performed during the same nights of the PNe observations.

\section{Results on Individual Objects}

The objects studied are presented in Table 1 together with the angular sizes that we have detected at the faintest flux level measured. The $\mathrm{H} \alpha+[\mathrm{N} \mathrm{II}]$ flux at the last closed contour level is given in column 4.

Table 1.

\begin{tabular}{|c|c|c|c|c|}
\hline Name & $\begin{array}{l}\text { H } \alpha+[N \text { I] } \\
\text { exposure } \\
\text { time (sec) }\end{array}$ & $\begin{array}{l}\text { [O III] } \\
\text { exposure } \\
\text { time (sec) }\end{array}$ & $\begin{array}{l}\text { Flux H }+ \text { [N II] } \\
10^{-17} \text { ergs } \\
\sec ^{-1} \mathrm{~cm}^{-2} \operatorname{arcsec}^{-2}\end{array}$ & $\begin{array}{l}\text { Angular } \\
\text { size } \\
\text { arcmin }\end{array}$ \\
\hline S176 & 11400 & & 1.4 & $12 \times 14$ \\
\hline S188 & 5520 & 7200 & 1.8 & $17 \times 13$ \\
\hline HFG1 & 10800 & 6300 & 0.7 & $17 \times 8$ \\
\hline IW1 & 18000 & 2700 & 0.6 & $10 \times 12$ \\
\hline A7 & 7800 & 4800 & 0.75 & $13 \times 13$ \\
\hline S68 & 1800 & 1800 & 3. & $15 \times 6$ \\
\hline A62 & 2700 & 1800 & 4. & $2 \times 3$ \\
\hline A74 & 8100 & 8100 & 0.9 & $14 \times 15$ \\
\hline IW2 & 9900 & & 1. & $16 \times 13$ \\
\hline
\end{tabular}




\section{Discussion}

We have presented results from our survey of extended, aged PNe in the solar neighborhood. A more extensive discussion as well as presentation of the complete PNe sample observed in our survey is presented elsewhere (Xilouris et al. 1993).

The sample of the 9 aged PNe that we have studied is characterized by asymmetric emission distribution, often disturbed by condensations which are evident in the $\mathrm{H} \alpha+[\mathrm{N} \mathrm{I}]$ image but vaguely seen in the [O III] high excitation stage. Generally the [O III] emission is confined near the center of the nebula while the $\mathrm{H} \alpha+[\mathrm{N} \mathrm{II}]$ distribution, often shocked, is detected at larger distances. In addition faint haloes which do not appear limb brightened are found at large distances in some PNe.

Disturbances in the emission of certain objects such as A74, S188, S68, HFG1, IW2, IW1, S176, as well as displacement of the central star indicate that those PNe are interacting with the ISM, in good agreement with the predictions of Borkowski et al. 1990. This interaction is partly responsible for their present shaping.

\section{References}

Balick, B., 1987. Astron. J., 94, 671.

Balick, B., Gonzalez, G., Frank, A. and Jacoby, G., 1992. Astrophys. J., 392, 582.

Borkowski, K.J., Sarazin, C.L. and Soker, N., 1990. Astrophys. J., 360, 173.

Chu, Y.-H., Jacoby, G.H. and Arendt, R., 1987. Astrophys. J. Suppl., 64, 529.

Ishida, K. and Weinberger, R., 1987. Astron. \& Astrophys., 178, 227.

Jacoby, G.H., 1981. Astrophys. J., 244, 903.

Weinberger, R., 1989. Astron. \& Astrophys. Suppl., 78, 301.

Xilouris, K.M., Paleologou, E. and Papamastorakis, J. To be submitted. 